\title{
Counteracting instabilities of photomultiplier tubes for multi-element detector systems
}

\author{
V. Astromskas, ${ }^{a,{ }^{*}}$ M. J. Joyce ${ }^{a}$ and M. Aspinall ${ }^{a}$ \\ ${ }^{a}$ Lancaster University, \\ Engineering Department, Lancaster University, Bailrigg, Lancaster, LA1 4YW, United Kingdom \\ E-mail: V.Astromskaselancaster.ac.uk
}

\begin{abstract}
This paper presents an investigation into response of instabilities of EJ-309 liquid scintillator detectors. A brief review of common instabilities associated with the photomultiplier tubes (PMTs) is presented. The energy response, relative efficiency, energy linearity and warmup duration of sixteen EJ-309 detectors coupled to PMTs tested is presented. A single-channel mixed-field analyser digitiser system was used for data acquisition. Furthermore, timing information of the common instability behaviours is presented alongside suggestions on how to correct for such instabilities. The results show that the relative efficiencies amongst the detectors can vary up to 7\%; a multi-energy energy calibration must be performed to ensure energy linearity; the detectors must be warmed-up by $\sim 45$ minutes before stable response is achieved; the re-warmup duration depends on the duration of the high voltage supplied to the PMT being switched off. The results indicate that the PMTs take approximately 2 hours to reach "cold" state, where a full warm-up duration must be applied. The reported instability effects will be taken into account when developing a sophisticated auto-calibration methodology for a multi-element detector system.
\end{abstract}

KEYWORDS: Scintillators, scintillation and light emission processes (solid, gas and liquid scintillators); Neutron detectors (cold, thermal, fast neutrons); Photon detectors for UV, visible and IR photons (vacuum) (photomultipliers, HPDs, others).

\footnotetext{
${ }^{*}$ Corresponding author.
} 


\section{Contents}

\section{Introduction}

Bookmark not defined.

2. Methods

\section{Results and Discussion}

3.1 Uniformity

3.2 Efficiency

3.3 Stability and Warm-up

\section{Introduction}

Multi-element scintillator systems are used increasingly in a broad range of applications such as neutron spectrometry [1], nuclear security [2-4], fundamental studies of nuclear reactions [5-7], and in medical imaging for positron emission tomography (PET), single-photon emission computed tomography (SPECT) or X-ray imaging [8]; in such applications it is usually required that the response of each detector across an array is as consistent as possible. Non-destructive active interrogation techniques utilising neutrons and photons operate in environments where the incoming radiation flux can change by a few orders of magnitude in an instant, thus requiring detection systems to have a characterised drift of response [9].

Typically, however, there can often be significant differences in the performance of the scintillator material, the photomultiplier tubes and/or the quality of the coupling between them and readout systems. This can lead to variance in detector system performance if it is not accounted for. This is especially important for materials accountancy applications associated with material identification and assessment based on the detection of radiation from mixed fields. For example, excess non-linearity or inconsistency with regards to energy response could lead to false identification or excess uncertainty in the assessment of a given nuclear material.

Normally, in such applications, the detectors are calibrated using laboratory-based sources of $\gamma$ radiation to account for differences in the linearity of energy response. Whilst effective, this method ignores the potential for there to be inconsistency in gain settings, which can be particularly important for multiplicity assay where the basis for the measurement is the coincident detection of neutrons (usually) in two or more detectors at effectively the same time. Given the ubiquity of multiplicity assay in practical exercises carried out for the benefit of non-proliferation treaty verification, it is clear that an improved knowledge of the stability of these systems is vital for real-world applications.

PMT manufacturers describe instabilities as the change of the constancy of the anode sensitivity with respect to time, temperature, mean current and environmental effects [10]. The two major departures from constancy are long-term drift and short-term drift which are described in detail by Flyckt and Marmonier [10]. The long-term drift is primarily comprised of high-current drift and low-current drift. The high-current drift is observed typically after a few months of inactivity and at mean anode currents exceeding $10 \mu \mathrm{A}$. It can be characterised as a large relative gain drift of $20 \%$ to $40 \%$ occurring for the first 24 to 48 hours of operation. Afterwards, the gain is relatively stable for thousands of hours of operation. The low-current drift describes the relative gain changes that occur when a PMT is switched off and is subjected to more or less constant illumination. Higher anode currents reduce the gain variation duration. The relative gain variation is different for different types of PMTs as well as amongst the same models which typically have a 5\% gain variation. The gain drift can be characterised by multiple behaviours which are displayed in figure 1 . The behaviours have different maximum gain drifts, as well as, different stabilisation durations which can vary between 12 and 24+ hours [10]. 


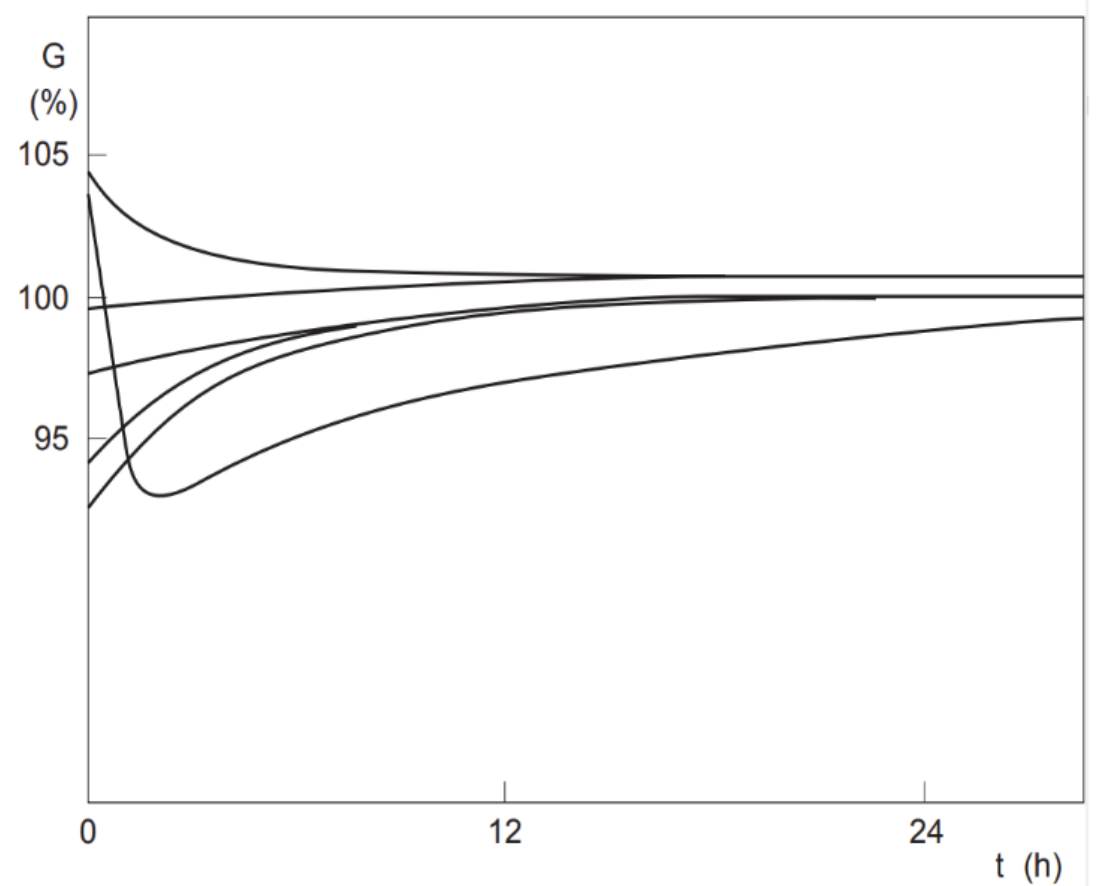

Figure 1. Examples of initial low-current drift behaviours represented in relative gain $G$ versus illumination time $t$ [10].

The short-term drift is to be considered when the PMT is exposed to a flux that gives rise to a mean anode current of less than $10 \mu \mathrm{A}$. In this case, the gain is typically stabilised after (10 to 15) minutes for the long-term drift effect to be disregarded. If the flux is changed abruptly, the anode current starts a new drift phase before stabilising once more which typically takes a few seconds. However, the new mean value of the anode current is often increased during this short-term drift resulting in hysteresis. Depending on the magnitude of the flux increase, the hysteresis could induce a relative gain drift of up to $0.5 \%$ [10].

This paper presents an investigation of the normalisation of the performance for a multi-element scintillator detector system. A part of this work has been presented at the ANIMMA 2017 conference and received positive engagement from the scintillation detector community [11].

\section{Methods}

The uniformity of response of sixteen EJ-309 [12] liquid scintillators of type VS-1105-21 (Scionix, Netherlands), coupled to photomultiplier tubes (PMTs) of type 9821 FLB (ADIT Electron Tubes, Sweetwater, TX) was tested with a number of $\gamma$ radiation sources. Each organic liquid scintillator unit is contained in a 3-mm thick aluminium chamber of dimensions $10 \mathrm{~cm} \times 10 \mathrm{~cm} \times 12 \mathrm{~cm}$. The PMTs used were $336 \mathrm{~mm}$ in length and $58.8 \mathrm{~mm}$ in diameter. The PMTs were connected to a single-channel, mixed-field analyser (MFA) digitiser system (Hybrid Instruments Ltd., UK) for signal processing and data readout [13]. The high voltage to the detectors was also supplied from the built-in HV supply in the MFA unit, ranging from -1546 V to -1973 V. The same MFA unit, HV supply, BNC and Ethernet cables were used for all experiments. Each detector was characterised individually in a laboratory with temperature stabilised with the pre-existing, installed domestic climate control system. The temperature fluctuations in the room were approximately $\pm 2{ }^{\circ} \mathrm{C}$. Not all 16 detectors were tested for every test reported in this work due to limited availability of all of the detectors for all of the tests throughout the experimental period. However, the behaviours reported in this paper were observed to affect all the detectors to a similar extent.

Sealed, laboratory-based radioactive sources, namely in this case, ${ }^{137} \mathrm{Cs},{ }^{22} \mathrm{Na}$ and ${ }^{60} \mathrm{Co}$ were used to evaluate the uniformity of the detector's energy sensitivity and relative efficiency. For all experiments, a radioactive source was placed at the centre on top of a detector held in the vertical plane. The detector was held in the same orientation for each of the radioactive sources tested. This prevented variations in the number of counts detected due to the air bubble in the enclosure moving and changing the contact between the scintillant liquid and the optical window [14].

Each of the detectors were calibrated by adjusting the high voltage in such a way that the Compton edge derived from the ${ }^{137} \mathrm{Cs}$ response would be centred, in this case at channel 200, on the MFA graphical user interface. The MFA was set to bin the data into 500 channels. 10-point moving filter was applied on the spectrum to help guiding 
the eye during calibration. The response exhibits the characteristic peak-shape before the drop-off, thus, enabling the use of a Gaussian fit on the Compton edge to evaluate the behaviour of the detectors. Given that EJ-309 scintillator has an anthracene crystalline structure, effectively, no photopeaks are observed as the low atomic number of the absorber makes Compton effect the principal interaction between $\gamma$ rays and EJ-309 [15]. Thus, the Compton edge was used to evaluate the behaviour of the detectors. The same HV level was then kept for ${ }^{22} \mathrm{Na}$ and ${ }^{60} \mathrm{Co}$ spectra in order to identify the disparities in energy linearity between different detectors.

For energy response and relative efficiency measurements, the spectra were collected for 10 minutes in the case of ${ }^{137} \mathrm{Cs}(330 \mathrm{kBq})$, and for 30 minutes for ${ }^{22} \mathrm{Na}(2.49 \mathrm{kBq})$ and ${ }^{60} \mathrm{Co}(8.93 \mathrm{kBq})$, with the difference in duration being made to accommodate the different activities of the sources available for the measurements. For warm-up duration evaluation, the spectra were collected continuously for 2 hours using ${ }^{137} \mathrm{Cs}$ source. Moreover, to investigate the effect of powering a detector off and its effect on performance, the same detector was exposed to ${ }^{137} \mathrm{Cs}$ source for 1 hour without interruption in order to achieve stable performance. Subsequently, the whole MFA unit was powered off which, in turn, switches off the HV supplied to the detector.

The same HV level was applied throughout the power cycles to maintain consistency across the calibration. The durations over which the power was cycled were $1 \mathrm{~min}, 5 \mathrm{~min}, 10 \mathrm{~min}, 20 \mathrm{~min}$, and $30 \mathrm{~min}$. Afterwards, the MFA unit was powered up with the same settings, configuration and HV supply level etc., and once again the detector was exposed to the ${ }^{137} \mathrm{Cs}$ source continuously for a further 1 hour. The HV stability measurements were performed by probing the HV cable connected between the HV supply of the MFAx1.3, Serial No. 1315 and the EJ-309 detector \#13. The probe was connected to an Agilent MSO810a oscilloscope which, in turn, was connected to a laptop via Ethernet connection, and the data was collected using a Matlab® script. First, the datum was collected continuously for 1 hour in order to measure the stability of the applied high voltage to the detector. The second measurement was conducted by power-cycling the applied high voltage while continuously measuring the applied high voltage. The "off" durations ranged from 10 seconds to 3 minutes.

Table 1. The model numbers of the photomultipliers and their corresponding detector reference numbers.

\begin{tabular}{|l|l|l|l|l|l|l|l|l|l|l|l|l|l|l|l|l|}
\hline Serial & SBM & SBM & SBM & SBM & SBM & SBM & SBM & SBM & SBM & SBM & SBM & SBM & SBM & SBM & SBM & SBM \\
Number & 435 & 436 & 824 & 831 & 843 & 847 & 850 & 828 & 832 & 840 & 835 & 838 & 827 & 839 & 854 & 829 \\
\hline $\begin{array}{l}\text { Detector } \\
\text { Number }\end{array}$ & 1 & 2 & 3 & 4 & 5 & 6 & 7 & 8 & 9 & 10 & 11 & 12 & 13 & 14 & 15 & 16 \\
\hline
\end{tabular}

\section{Results and Discussion}

\subsection{Uniformity}

Figure 2 represents the energy response of seven of the EJ-309 detectors in terms of the peak position of the Compton edge. The data were extracted via a Gaussian fit to the Compton edge in the response obtained for each of the radioactive sources. A single Gaussian fit was used for the ${ }^{60} \mathrm{Co} 1173 \mathrm{keV}$ and $1332 \mathrm{keV}$ Compton edge, since the energy resolution of the detector was too poor to distinguish the peaks. The detectors were calibrated relative to the ${ }^{137} \mathrm{Cs}$ response. The error bars were estimated on the basis of each of the fits and are generally smaller than the symbol used to indicate the position of each datum. As shown in the figure, there is a greater deviation between the resolved channel number in the higher-energy channels compared to the lower energy ones. The resolved peak position of the Compton edge amongst the seven detectors differed by $9 \%$ for $511 \mathrm{keV}, 2 \%$ for $662 \mathrm{keV}, 15 \%$ for $1173 \mathrm{keV}$, and $14 \%$ for $1275 \mathrm{keV}$.

The residuals of the linear fit of the energy response of seven detectors are shown in figure 3 . The lines associated with each data point are for eye-guiding purposes. The slopes of the linear fits extracted from data vary significantly by up to $18 \%$. The standard deviation of the data is $\sim 5 \%$. The errors of the peak positions extracted from the Gaussian fit were on average $1.1 \%$ for $511 \mathrm{keV}, 0.2 \%$ for $662 \mathrm{keV}, 0.1 \%$ for $1173 \mathrm{keV}$, and $0.7 \%$ for $1275 \mathrm{keV}$ peak positions. The extracted R-Square values from the linear fits ranged from 0.871 to 0.927 . The 
extracted Chi-squared values ranged from 4.41 to 524 amongst the seven detectors, thus, proving that the response in terms of energy is not linear. These results suggest that energy calibration must be performed to ensure that the multi-element system energy response is uniform. Moreover, these results imply that the energy calibration cannot be performed using a single energy as the reported energy non-linearity may affect each detector differently. Thus, an energy linearity calibration using multiple energies ought to be included in the calibration methodology to normalise the energy response.

Figure 2. Peak position (channel number) versus detector number to give the energy response of seven EJ-309 detectors for ${ }^{137} \mathrm{Cs}$, ${ }^{22} \mathrm{Na}$ and ${ }^{60} \mathrm{Co}$ radioactive sources. The peak positions were extracted using a Gaussian fit on the Compton edge of the aforementioned sources.

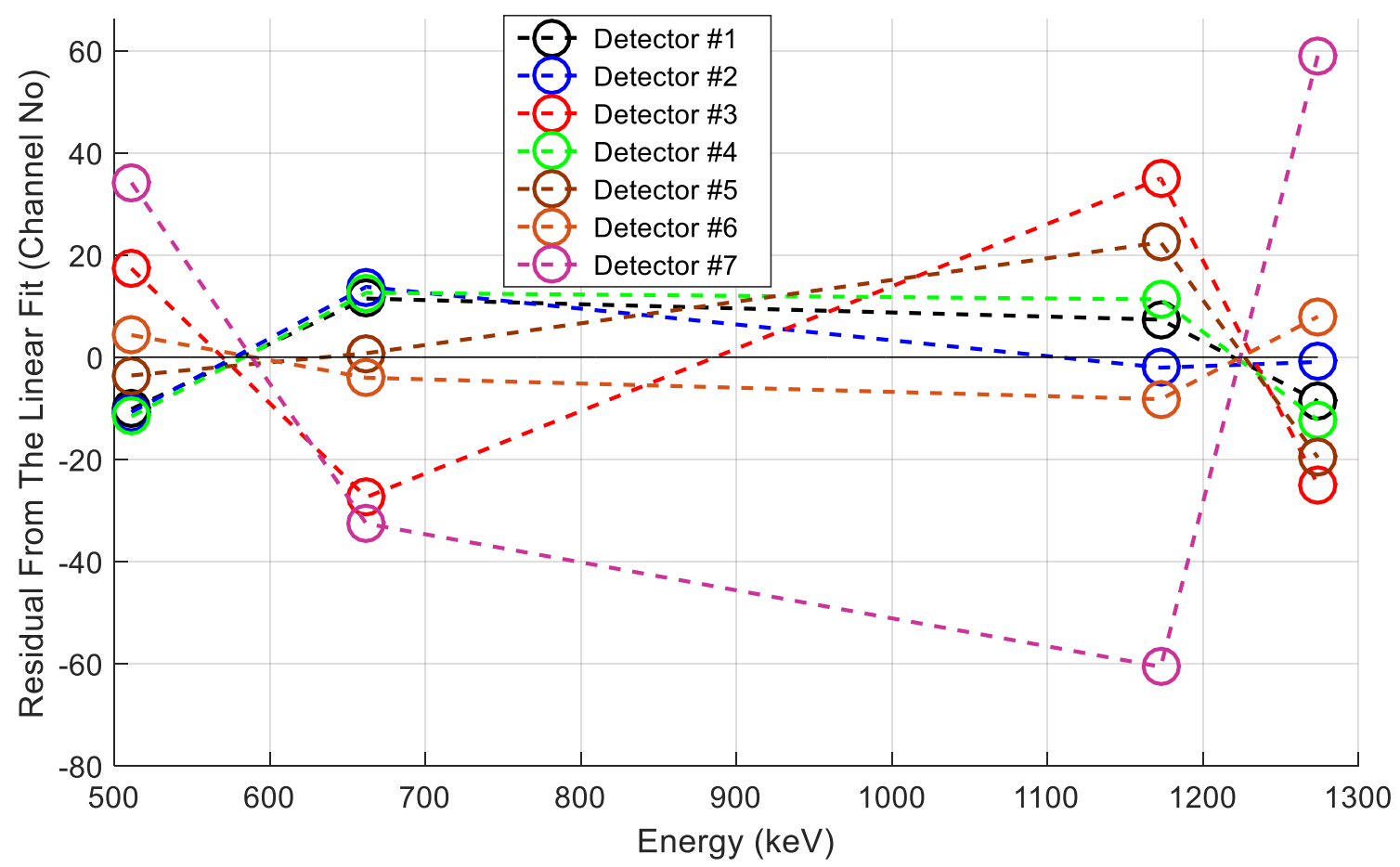

Figure 3. Residual of the linear fit of the peak position (i.e. channel number) versus energy (keV) for each of the seven detectors to infer the non-linearity of the response in terms of energy. The lines between the data points are for eye-guidance. 


\subsection{Efficiency}

The normalised relative efficiency of the seven detectors is shown in figure 4. The efficiency was obtained by summing the events from each of the spectra for each detector and normalising this number to the number of events recorded by the detector with the highest number of counts. The error bars were obtained by error propagation of the error from the Gaussian fit and the statistical error. In figure 4 detector number 5 gave the response that the other detectors were normalised to. Given that the data were collected for the same amount of time and with the same radioactive source in the same position, figure 4 depicts the variation in relative detection efficiency. For the majority of the cases, the efficiencies varied by up to $7 \%$. However, in one particular case the relative efficiency was $23 \%$ less than that compared to the other detectors. The great variation of gain of the ${ }^{137} \mathrm{Cs}$ response of detector 6 could have been a spurious effect. However, the measurement was not repeated due to inability to access the detector for additional measurements. No data were excluded from the analysis. Interestingly, the applied high voltage was identical for detectors 1 and 6 while showing the greatest difference in efficiency, especially in the case of ${ }^{22} \mathrm{Na}$.

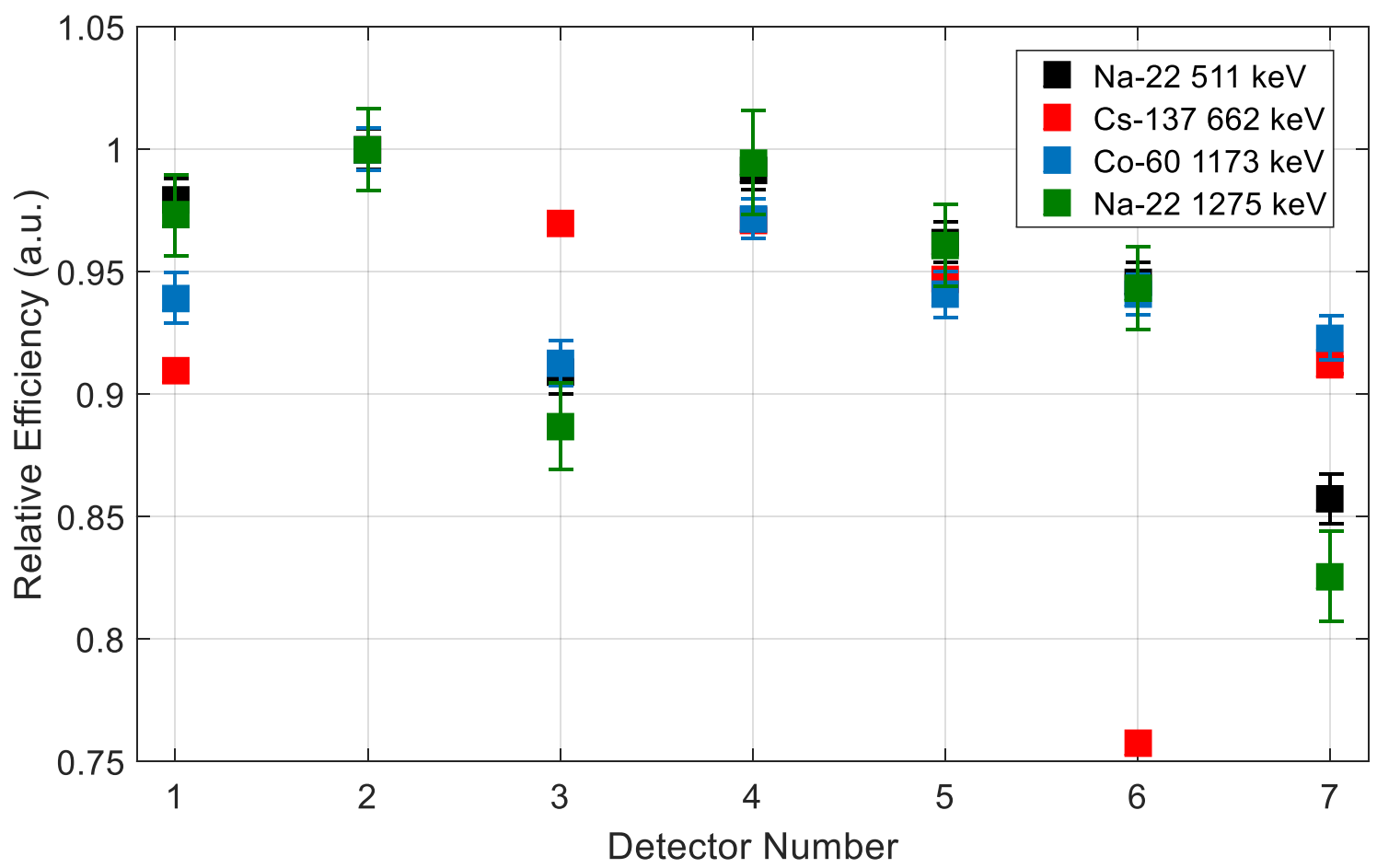

Figure 4. Normalised relative efficiency (a.u.) of the seven tested EJ-309 detectors versus detector number. The efficiency is expressed normalised relative to the detector with the highest counts in the ${ }^{60} \mathrm{Co},{ }^{137} \mathrm{Cs}$ and ${ }^{22} \mathrm{Na}$ Compton edge peaks (in this case detector 5).

The relative efficiencies can be corrected by a first-order correction factor that will be different for each element when calibrating a multi-element detector system, as inferred by the data in figure 4 . To maintain data integrity, i.e. to only have the real measured counts, it is suggested that a correction factor of $<1$ is used in order to normalise the multi-element array to the least efficient detector. This would result in excluding the data from the more efficient detectors to achieve normality of response amongst the array, i.e. "a chain is only as strong as its weakest link." A correction factor of $>1$, i.e. normalising to the most efficient detector, would results in a proportion of the counts being effectively fabricated. This approach ensures that all of the measured events will be correct but at the cost of overall efficiency. 


\subsection{Stability and Warm-Up}

Firstly, as shown in figure 5, most of the detectors exhibited a stable performance after a warm-up period had elapsed as depicted by the steady state region. The data were obtained from sampling the 2-hour data every 2 minutes. The data in figure 5 suggest that the calibration between the detectors was not perfect, thus resulting in minor variation of approximately 10 channels of the centroid of the peak throughout the measurement.

Figure 6 displays the effect of the warm-up period on the standard deviation of the fitted Gaussian peak of the Compton edge, which is linked directly to FWHM of energy resolution of the detector. A relative increase of approximately $(4 \pm 2) \%$ was measured for all detectors. Also, the majority of the tested detectors had similar standard deviations with two of them deviating by \pm 10 channels. This behaviour was observed repeatedly over multiple tests.

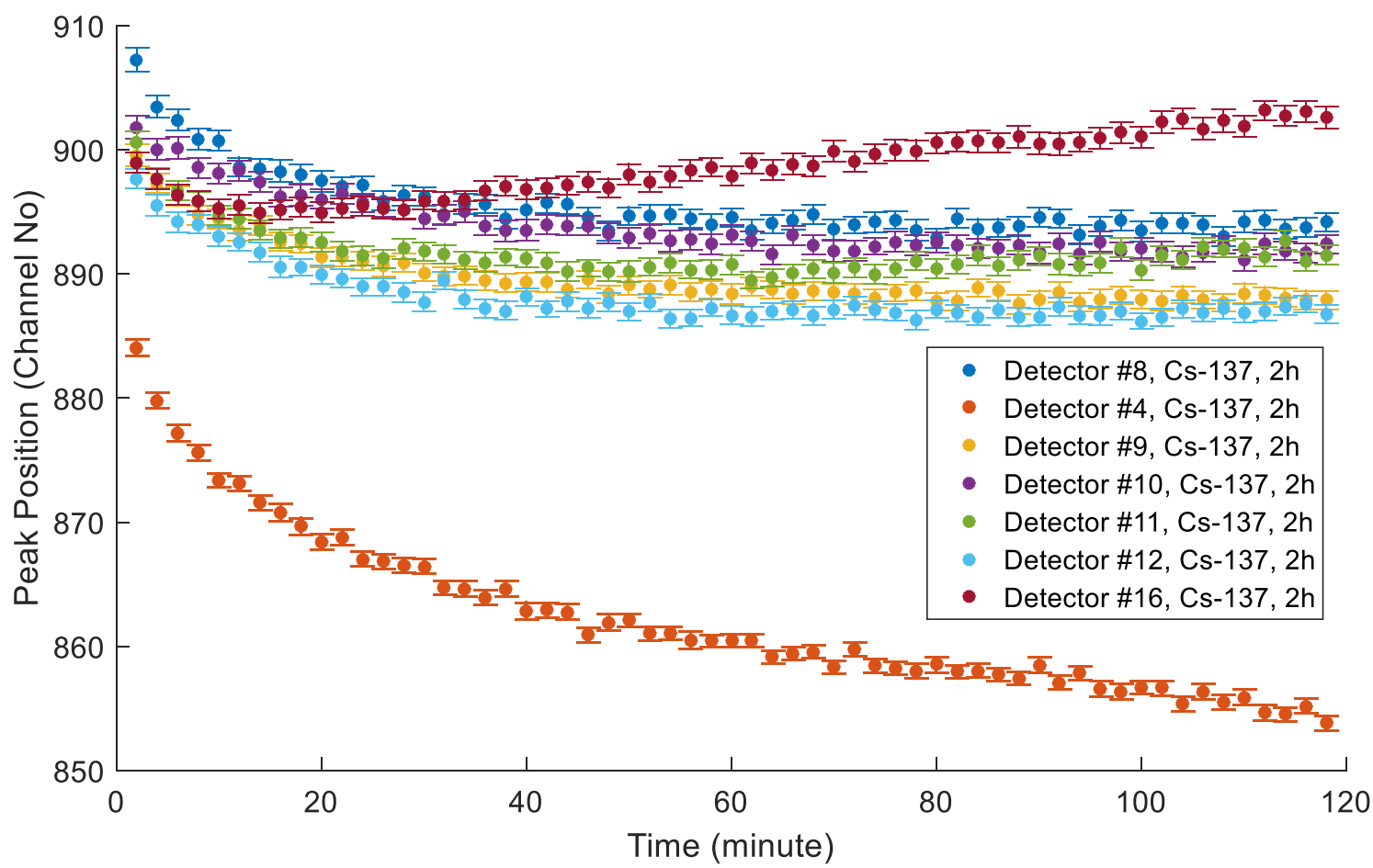

Figure 5. Peak position (channel number) versus time (minute), showing the evolution of the extracted centroid of the Compton edge from the Gaussian fit of the ${ }^{137} \mathrm{Cs}$ response over 2 hours and for 7 different detectors.

These experiments revealed three typical behaviours of the detectors as depicted in figure 7. Firstly, the most common behaviour is represented by the red line, where for the first 45 minutes the detector is warming up and stabilising, resulting in the shift of the centroid of the Compton edge towards lower energy channels by (10 to 20) channels. This is followed by a steady state region where a detector's response is considered stable. The second behaviour is displayed by the blue line, where performance is approximately stable throughout the experiment. Third, the most dynamic behaviour follows the trend depicted by the green line, where the resolved peak position of the Compton edge is moving towards higher-energy channels without reaching a stable performance. After 2 hours of continuous exposure, the detector had not stabilised.

It is worth noting that the detectors with the behaviour displayed in figure 7 had recently been returned from maintenance prior to these tests. Detectors \#14 and \#15 had their potentiometer replaced, while detector \#13 had no work done. The source of the unstable behaviour is currently unidentified as this behaviour was observed in only 3 of the 16 tested detectors using the exact same setup and over multiple measurements and power cycles. It suggests that the source of the issue is inside the PMT and agrees with the possible photomultiplier long-term gain drift behaviours reported by the literature [9]. 
Figure 6. The standard deviation of the fit to the Compton edge versus time (minute), demonstrating the evolution of the extracted standard deviation from the Gaussian fit of the ${ }^{137} \mathrm{Cs}$ Compton edge over 2 hours for 7 different detectors.

Figure 7. Peak position (channel number) versus time (minute) depicting three distinct behaviours of the drift of the peak position of the Gaussian fit on the ${ }^{137} \mathrm{Cs}$ Compton edge.

These significantly different behaviours suggest that calibration should be performed in two stages for any photomultiplier tube. First, a constant exposure for (30 to 40) minutes would identify which behaviours the characterised detector is exhibiting. Second, identify the stabilisation duration associated with each behaviour by 
continuing constant illumination until the behaviour stabilises which can vary significantly depending on the size and specifications of the photomultiplier tube. This two-staged calibration would allow detectors with the same instability in the multi-element system to be grouped together and ensure that the same stabilisation duration is required to reach the constancy those detectors.

Figure 8. Peak position (channel numbers) versus time (minute) showing the evolution of peak position over a 1-hour exposure after $10 \mathrm{~min}, 20 \mathrm{~min}$ and $30 \mathrm{~min}$ period with the power off.

Figure 9. Applied high voltage (V) versus time (s) showing the stability of the applied high voltage to a EJ-309 detector over 1 hour. The errors bars correspond to 3 standard deviations.

Figure 8 shows the effect of powering off the detector system on the warm-up duration. Each data point represents a 2-minute sample of a continuous 60-minute measurement. The data were fitted with an empirical, doubleexponential fit in order to visualise the trend. The first deviation from stable operation was observed after the 
detectors were powered off for 10 minutes. The warm-up duration after 10 minutes with the power off required to stabilise the detector was approximately 5 minutes. As the power-off duration was increased, the warm-up duration before reaching stable performance also increased. After a 30-minute power-off period, the stable performance was achieved after $\sim 12$ minutes. Extrapolating the data, a full detector warm-up cycle of approximately 45 minutes ought to be applied after the detector was powered off for approximately (90 to 120) minutes in order to achieve the stable performance once again. Moreover, a difference of up to $1 \%$ in the resolved peak position is observed in figure 8 in between the cycles. This suggests that the calibration is not identical for every power cycle and that the PMT requires a new calibration after every power cycle, given that the variation in the HV supply was approximately $\pm 2 \mathrm{~V}$ as shown in figures 9 and 10 . The drift and variations of the applied high voltage of $\pm 0.5 \mathrm{~V}$ are negligible in comparison to the intrinsic variation of $1 \%$ of the applied high voltage, i.e. $\pm 2 \mathrm{~V}$. Moreover, the applied high voltage is stable after switching of the high voltage as shown in figure 10. Thus, the warm-up cycle must be included into the auto-calibration of the multi-element detector system methodology to ensure a stable performance of all detectors.

Figure 10. Applied high voltage (V) versus time (s) displaying the stable applied high voltage after switching the high voltage supply on and off. The "off" durations ranged from $10 \mathrm{~s}$ to $3 \mathrm{~min}$. The error bars correspond to 3 standard deviations.

Furthermore, the warm-up duration for two EJ-301 detectors coupled to a smaller PMTs was approximately 15 minutes, three times faster than the larger EJ-309 PMTs. The EJ-301 scintillants were coupled to a Hamamatsu 3/4" Type 5611 PMTs which had the following dimensions: $85 \mathrm{~mm}$ in length and $35 \mathrm{~mm}$ in diameter. This suggests that the warm-up duration is potentially dependent on the size, dynode geometry and the material of the PMT which needs to be investigated in future.

\section{Summary}

Sixteen EJ-309 liquid scintillator detectors have been tested to study their stability, energy response and warm-up requirements. The results from these tests indicated that:

- The relative efficiency of the detectors can vary on average by up to 7\%,

- An energy calibration based on a single line (peak) does not produce a linear energy response over energies ranging from $511 \mathrm{keV}$ to $1275 \mathrm{keV}$,

- The detectors on average require $(40 \pm 5)$ minute to allow their initial response to stabilise. 
- Three distinct behaviours of EJ-309 detectors are reported, one of which is unstable.

- The detectors take on average 2 hours before reaching a "cold" state after being switched off, where a full warm-up duration must be applied.

This research summarises a number of effects that ought to be taken into account when calibrating a multi-element detector system and provides estimates as to the extent of these effects. The instability effects reported in this research fall under the photomultiplier tube long-term gain drift category, thus, indicating that these effects must be taken into account by the scintillator community. This research suggests that extensive details of multi-element detector system calibration when publishing results from a multi-element arrays are desirable in order to compare and evaluate the accuracy of the calibration methodology employed. Future research will concentrate on a development of a uniform multi-element system calibration methodology which will take all of the aforementioned effects into account. Furthermore, in future work 42 detectors will be fully tested in terms of their instabilities.

\section{Acknowledgements}

We acknowledge the support of the Engineering and Physical Sciences Research Council, under grant EP/M02489X/1, and via the ADRIANA grant as part of the UK National Nuclear User Facility (NNUF), under grant EP/L025672/1. M. J. J. acknowledges the support of the Royal Society via a Wolfson Research Merit Award.

\section{References}

[1] R. Machrafi, A. L. Miller, and N. Khan, "New approach to neutron spectrometry with multi element scintillator" Radiation Measurements, vol. 80, pp. 10-16, Sept. 2015.

[2] G. Popescu, S. Herman, S. Glover, and H. Spitz, “Compton background suppression with a multi-element scintillation detector using high speed data acquisition and digital signal processing," Journal of Radioanalytical and Nuclear Chemistry, vol. 307, pp. 1949-1955, Mar. 2016.

[3] S. A. Pozzi, M. C. Hamel, K. Polack, M. J. Marcath, T. H. Shin, A. Di Fulvio, and S. D. Clarke. "Detection for Nuclear Nonproliferation," Proc. Int. Symp. on Radiation Detectors and Their Uses (ISRD2016), JPS Conf. Proc. $11,050001,2016$.

[4] Jennifer L. Dolan, Marek Flaska, Alexis Poitrasson-Riviere, Andreas Enqvist, Paolo Peerani, David L. Chichester, Sara A. Pozzi, "Plutonium Measurements with a fast-neutron multiplicity counter for nuclear Safeguards Applications" Nucl. Instr. Meth. A, 763, (2014), pp 565-574

[5] M. Febbraro, F. D. Becchetti, R. O. Torres-Isea, M. Ojaruega, A. M. Howard, J. J. Kolata, A. Roberts, and A. N. Villano, "Neutron Spectroscopy Without Time-of-Flight Measurement: A DSP-Based Deuterated Scintillator Array,” IEEE Transactions on Nuclear Science, vol. 60, pp. 890-896, Apr. 2013.

[6] Jonathan S. Beaumont, B. Alan Shippen, Matthew P. Mellor, and Malcolm J. Joyce. "Imaging of Fast Neutrons and Gamma Rays from 252Cf in a Heavily Shielded Environment." Nuclear Instruments and Methods in Physics Research Section A: Accelerators, Spectrometers, Detectors and Associated Equipment 847 (March 1, 2017): 77-85. https://doi.org/10.1016/j.nima.2016.11.043.

[7] Parker, Helen M. O., Michael D. Aspinall, Alex Couture, Francis D. Cave, Christopher Orr, Bryan Swinson, and Malcolm J. Joyce. "Active Fast Neutron Singles Assay of 235U Enrichment in Small Samples of Triuranium Octoxide.” Progress in Nuclear Energy 93 (November 1, 2016): 59-66. https://doi.org/10.1016/j.pnucene.2016.07.022.

[8] Paul Lecoq. "Development of New Scintillators for Medical Applications." Nuclear Instruments and Methods in Physics Research Section A: Accelerators, Spectrometers, Detectors and Associated Equipment, Advances in detectors and applications for medicine, 809 (February 11, 2016): 130-39. https://doi.org/10.1016/j.nima.2015.08.041.

[9] Jones, A. R., M. D. Aspinall, and M. J. Joyce. “A Remotely Triggered Fast Neutron Detection Instrument Based on a Plastic Organic Scintillator.” Review of Scientific Instruments 89, no. 2 (February 2018): 023115. https://doi.org/10.1063/1.5012121. 
[10] S-O Flyckt, and Carole Marmonier. Photomultiplier Tubes: Principles \& Applications, 2002.

[11] V. Astromskas, and M. J. Joyce. "Time-Dependent Characterisation of Stability Performance of EJ-309 Detector Systems.” EPJ Web of Conferences 170 (2018): 01002. https://doi.org/10.1051/epjconf/201817001002.

[12] "Eljen Technology - EJ-301, EJ-309." [Online]. Available: https://eljentechnology.com/products/liquidscintillators/ej-301-ej-309. [Accessed: 16-May-2018].

[13] M. J. Joyce, M. D. Aspinall, F. D. Cave, K. Georgopoulos, and Z. Jarrah, "The design, build and test of a digital analyzer for mixed radiation fields," IEEE Transactions on Nuclear Science, vol. 57 (5), pp. 2625-2630, 2010.

[14] A.R. Jones, and M.J. Joyce. "The Angular Dependence of Pulse Shape Discrimination and Detection Sensitivity in Cylindrical and Cubic EJ-309 Organic Liquid Scintillators." Journal of Instrumentation 12, no. 01 (January 17, 2017): T01005-T01005. https://doi.org/10.1088/1748-0221/12/01/T01005.

[15] G. R. Choppin, G. R. Choppin, J.-O. Liljenzin, and J. Rydberg, Radiochemistry and nuclear chemistry, 3rd ed. Woburn, MA: Butterworth-Heinemann, 2002. 